\title{
Correction: Role of Virtual Reality in Balance Training in Patients with Spinal Cord Injury: A Prospective Comparative Pre-Post Study
}

Madhusree Sengupta ${ }^{1}$, Anupam Gupta ${ }^{1}$, Meeka Khanna ${ }^{1}$, U. K. Rashmi Krishnan ${ }^{1}$, Dhritiman Chakrabarti ${ }^{2}$

${ }^{1}$ Department of Neurological Rehabilitation, National Institute of Mental Health and Neurosciences, Bangalore, India

${ }^{2}$ Department of Neuro Anaesthesia, National Institute of Mental Health and Neurosciences, Bangalore, India

Correction for the headings of Fig. 1 is needed. There was a mistake in the publishing process. So, we want to correct the headings of Fig. 1 to more appropriate terms, such as "allocation," "follow-up," and "analysis," respectively. The revised Fig. 1 is as follows.

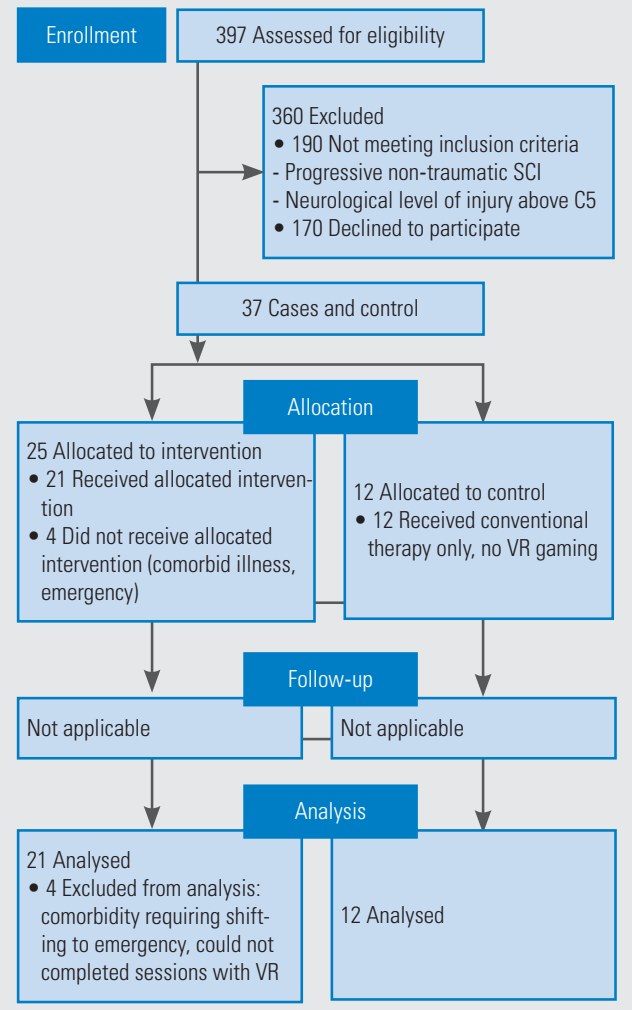

Fig. 1. Study design and flow chart. SCl, spinal cord injury; VR, virtual reality.

We apologize for any inconvenience that this may have caused. 